\title{
User-Optimal and System-Optimal Route Choices for a Large Road Network
}

\author{
DAVID BOYCE* \\ Northwestern University \\ QIAN XIONG
}

TJKM Transportation Consultants

\begin{abstract}
Solutions to the route choice problem for assumptions of user-optimality and system-optimality are presented for the road network of the Chicago region. Regionwide results show a $5 \%$ decrease in total travel time would be achieved during the morning peak period, if a system-optimal solution based on travel times were implemented. Among the costs of this solution is a $1.5 \%$ increase in vehicle-miles travelled. Findings for differences in link flows and individual origin-destination pairs complete the paper.
\end{abstract}

\section{Introduction}

Road pricing theory and analysis suggest that savings in travel time and related resources could be achieved if drivers were directed to travel along minimum marginal time routes. Equivalently, tolls based on the difference between marginal and average travel times might be used to induce drivers to shift to routes corresponding closer to a societal optimal. This theory has been well known since Beckmann et al. (1956); see Small (1992) and McDonald et al. (1999) for recent findings and syntheses of the road pricing literature.

The objective of this paper is to seek answers to questions related to the magnitude and distribution of these travel time savings in a large, congested urban road network. Obtaining such answers depends upon a capability to determine the route choices under two alternative criteria suggested by Wardrop (1952) and Beckmann et al. (1956):

- Drivers individually choose to follow their shortest time routes from their origins to destinations, leading to the situation that all used routes have equal travel time and no unused route has a lower travel time. Such a route pattern is called useroptimal or user-equilibrium, since it corresponds to a Nash equilibrium.

\footnotetext{
* Contact author: David Boyce, 2149 Grey Ave., Evanston, IL 60201 USA; Email: dboyce@uic.edu The findings presented here are based on Xiong (2002) and related research conducted at the University of Illinois at Chicago. We are grateful to Hillel Bar-Gera and Frederik Nöth for algorithmic and computational support, without which this study could not have been undertaken.
} 
- Drivers are centrally directed to use routes that result in the total travel time being minimized. This route pattern is called system-optimal.

In the early 1970s, convergent methods were first proposed to solve these two problems computationally for large networks. They have been routinely applied in urban transportation planning for the past 25 years. Until recently, however, those methods could not achieve sufficiently precise answers to conclude whether or not the observed differences in total travel times resulted from the route patterns or from computational errors. Following the invention of the Origin-Based Assignment (OBA) algorithm by BarGera (2002), such precision is now possible; see Patriksson (1994) for an overview of these models and solution methods.

The findings of the comparison of user-optimal and system-optimal route patterns presented below may be surprising as well as informative. The results provide insights into winners and losers as well as the aggregate savings, for both the Chicago region and individual travellers. The paper is organized as follows. First, a very short overview of the problem is given. Following an overview of the data, aggregate results are described. Then, maps of illustrative routes of travel from origins to destinations are presented. Discussions of limitations of the analysis and future research conclude the paper.

\section{$2 \quad$ Method}

The assumptions of the standard user-optimal route choice problem are relatively simple and idealized, but appear to provide useful information concerning route choices in reality. They may be summarized as follows:

- Drivers have perfect information concerning their shortest routes from origins to destinations. While the source of this information is not specified, we may presume it is from their past experience. The extent to which such an assumption is satisfied is unknown, as there is no large-scale data base on actual patterns of route choices.

- $\quad$ The problem is solved for fixed origin-destination (OD) flows (demands) over a relatively long time period, such as an hour or a peak period; during this period the flows are regarded as constant. Such models are termed static to contrast them with dynamic models in which the time period is a few minutes or even less. The flows are real-valued, and may be divided among more than one route.

- Origins and destinations of travellers are clustered into small zones. For the data used here, these zones vary in size from $1 / 16^{\text {th }}$ square mile to 36 square miles; however, the majority of the zones are approximately one square mile in area.

- Individual driver's travel times on road segments, called links, increase without limit with total link flow; in the standard model, these travel time-flow functions are separable, meaning they depend only on the link's own flow, and not the flow of conflicting links. This limitation can be relaxed, but generally is not in applications of such methods. 
- Choice of route by drivers is assumed to depend only on travel time, ignoring travel distance and tolls; an alternative assumption is that route choice is based on a linear combination of such variables, but only time is used here.

Given a representation of the road network in terms of intersections and road segments (nodes and links), a fixed matrix of OD flows, and the parameters of the link travel timeflow function (free flow travel time and a nominal capacity), the user-optimal (UO) and system-optimal (SO) patterns of route and link flows on the network can be found with great precision. For example, the maximum difference over all OD pairs between the minimum OD travel time and any other used route is less than $10^{-11}$ minutes.

For the user-optimal solution, for each OD pair drivers of all used routes experience the same travel times, and no unused route has a lower travel time. For the SO solution, the routes to which drivers are assigned have equal marginal travel times, and no unused route has a lower marginal travel time. If tolls were used to make drivers aware of the difference between the marginal and average route times, and all drivers responded to the tolls equally, then for all used routes the sum of travel time and tolls would be equal for each OD pair. In the SO case, the route travel times experienced by drivers for a given OD pair are different. Therefore, we computed the flow-weighted mean travel time from the SO routes for each OD pair for comparison with the UO travel time. Clearly, these solutions are idealized and simplified from reality.

\section{Overall findings}

An origin-destination (OD) trip matrix with one class of travellers was synthesized from a more comprehensive travel choice model of the Chicago Region. In this model, origindestination, mode and auto route choices are the solution variables of a large-scale, nonlinear optimization problem defined on a road network, a zone system and fixed matrices of OD travel times and fares for transit. Parameters of the model were estimated from a household travel survey undertaken during 1989-1991 by 17,000 households. Implementation, estimation and validation of the model with 1990 Census data is described by Boyce and Bar-Gera (2003).

\begin{tabular}{|c|r|r|r|r|r|r|r|}
\hline Flow - $\min$ & 0 & 0.005 & 0.1 & 1 & 10 & 100 & $>1,000$ \\
\hline $\max$ & $0.005^{1}$ & 0.1 & 1 & 10 & 100 & 1,000 & \\
\hline Number & 838,443 & $1,177,566$ & 829,665 & 275,000 & 13,902 & 92 & 2 \\
\hline
\end{tabular}

Table 1: Frequency distribution of origin-destination flows (vehicles per hour)

${ }^{1}$ Values less than 0.005 vehicles per hour were rounded to zero.

\begin{tabular}{|l|r|r|r|}
\hline \multicolumn{1}{|c|}{ Measure } & \multicolumn{1}{c|}{ User-optimal } & System-optimal & \multicolumn{1}{c|}{ Change (\%) } \\
\hline Vehicle-hours travelled & 564,012 & 535,909 & -5.0 \\
\hline Vehicle-miles travelled & $18,154,376$ & $18,424,776$ & +1.5 \\
\hline Space-mean-speed & 32.2 & 34.4 & +6.8 \\
\hline
\end{tabular}

Table 2: Network performance for user-optimal and system-optimal solutions 
The total flow in this trip matrix, which represents the morning peak period from 6:30 to $8: 30 \mathrm{am}$, is $1,360,427$ vehicles per hour. Because the trip matrix is synthesized from a continuous, real-valued model, it contains small flows of substantially less than one vehicle per hour between many OD pairs. Values of less than 0.005 vehicles per hour were rounded to zero. The number of OD pairs connecting the 1,778 internal zones is 3,134,670. The frequency distribution of these flows is shown in Table 1. For the solution of the UO and SO problems described in this paper, this trip matrix is a fixed input.

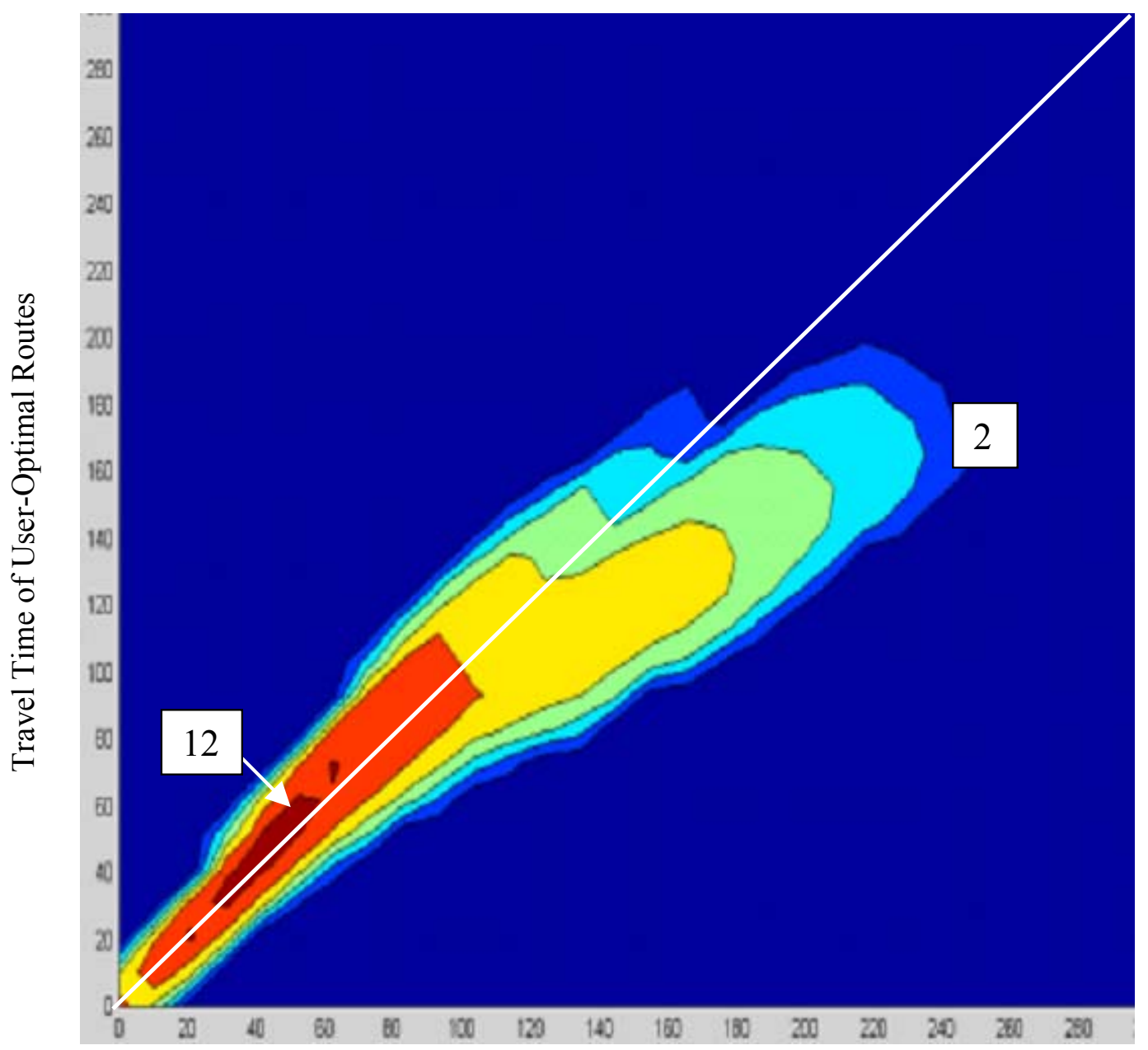

Mean Travel Time of System-Optimal Routes

\section{Figure 1: Contour map of OD user-optimal vs. system-optimal travel times}

Contour line:

No. of OD pairs:
4

55

6

403
8

2,980
10

22,026
12

162,755 
The road network used in this analysis consists of 12,982 nodes and 39,018 one-way links. The solution for the user-optimal model has 4,000 links with flow greater than the nominal capacity. Hence, we may conclude that this network is moderately congested.

Perhaps the first and foremost question concerns how much travel time is saved by the system-optimal solution, as compared with the user-optimal one. The answers to this and related questions pertaining to travel distance and speeds are shown next in Table 2.

As shown, the total travel time of the system-optimal solution is $5.0 \%$ less than for the user-optimal solution, but has additional travel distance of $1.5 \%$. The space-mean-speed, the ratio of distance travelled to time travelled, therefore increases by nearly $6.8 \%$, since distance increases while time falls. These results may be regarded as rather precise, given the assumptions. The time saving per vehicle is 1.2 minutes, whereas the additional distance travelled per vehicle is 0.20 miles. These results would increase, or decrease, for more or less congested systems.

Next, we consider how the travel times of individual OD pairs vary over the entire zone system. For each zone pair, we plot the UO travel time, which is identical for all used routes, vs. the flow-weighted mean of the SO route travel times, and form a contour map of the result, as shown in Figure 1. The axes of the figure are the UO and SO travel times in minutes. The contours show the natural logarithm of the number of OD pairs (unweighted by flow) in each 10 by 10 minute square in the plot area. The dark red area with the densest number of OD pairs has nearly 163,000 pairs per 10 by 10 minute square. The outer blue contour area has only 7 OD pairs in each 10 by 10 minute square. OD pairs lying above the $45^{\circ}$ line have UO times greater than mean SO times. The opposite is true for OD pairs lying below the line.

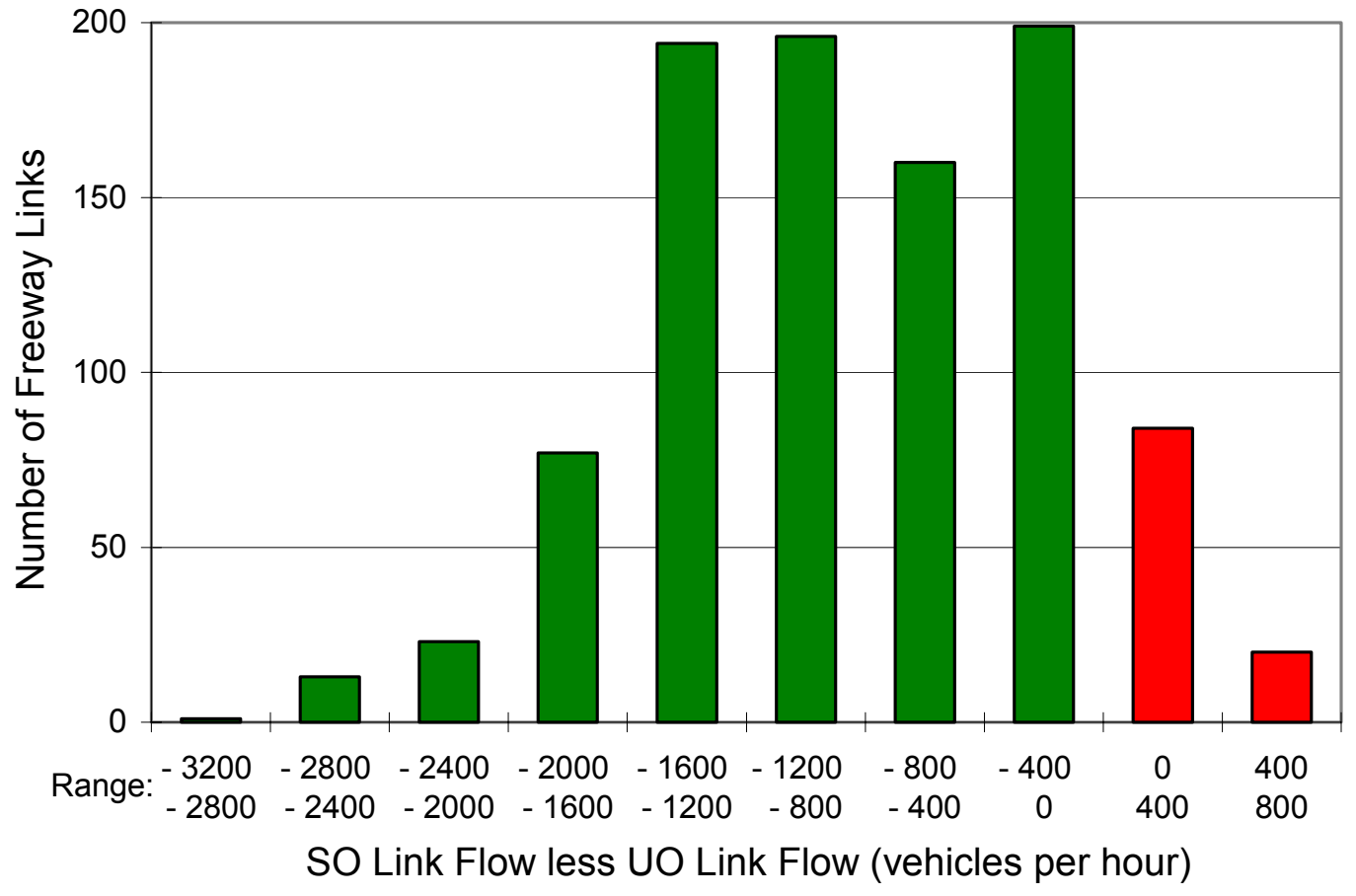

Figure 2: Frequency distribution of freeway link flow differences 
If the travel time is small ( 0 to 20 minutes), the contour plot shows the SO travel times are similar to the UO ones. For travel times between 20 and 60 minutes, where the highest density of OD pairs are found, roughly equal numbers of OD pairs have UO travel times greater than travel times and vice versa, as shown by the symmetry around the 45 degree line. For higher travel times, OD pairs with higher SO times than UO times (below the line) begin to dominate, but the number of these OD pairs is small. Careful examination of the figure provides insights into how many OD pairs would win and how many would lose if SO controls or incentives were implemented.

The figure may appear to emphasize excessively the more widely separated OD pairs with higher travel times, especially when one considers that the mean travel times are 24.9 minutes for user-optimal flows and 23.6 minutes for system-optimal flows. The OD pairs with lower travel times have higher OD flows, so when the flow-weighted times are computed, the means are small compared to the range of travel times of 0 to 200 minutes for the UO solution vs. 0 to 250 minutes for SO solution.

The two solutions may also be compared by examining the frequency distribution of the differences in link flows between the UO and SO solutions. Figures 2 and 3 show the frequency distributions of SO link flows less UO link flows for freeway links and arterial links respectively. Of the 976 freeway links (including expressways and tollways), 104 have larger SO flows than UO flows, and 872 links have lower SO flows, showing that flows are shifted from freeway links to arterial links in the SO solution. As shown in Figure 2, most freeway links have from 0 to 1,600 fewer vehicles per hour in the SO solution. Of the 34,359 arterial links, 22,159 links, or about $65 \%$, have larger SO flows than UO flows. However, the changes in arterial link flows are quite symmetrical, but shifted to the positive flow differences, as shown in Figure 3.

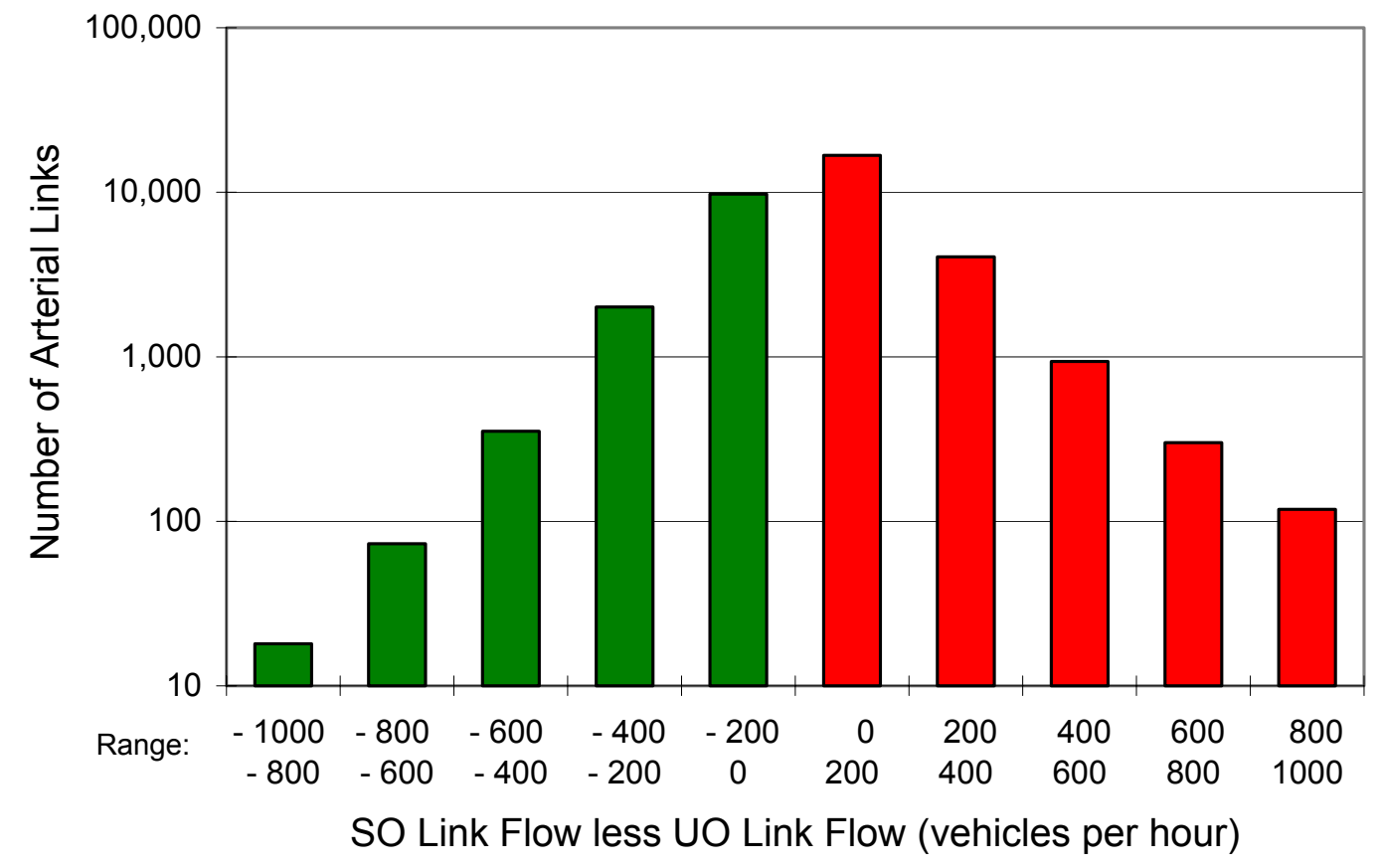

Figure 3: Frequency distribution of arterial link flow differences 


\section{$4 \quad$ Origin-destination specific findings}

Next, we consider examples of routes for selected origin-destination pairs. The objective here is to illustrate how certain OD pairs can have very different solutions for SO flows as compared with UO ones. Moreover, we show for one OD pair that the number of routes is relatively large.

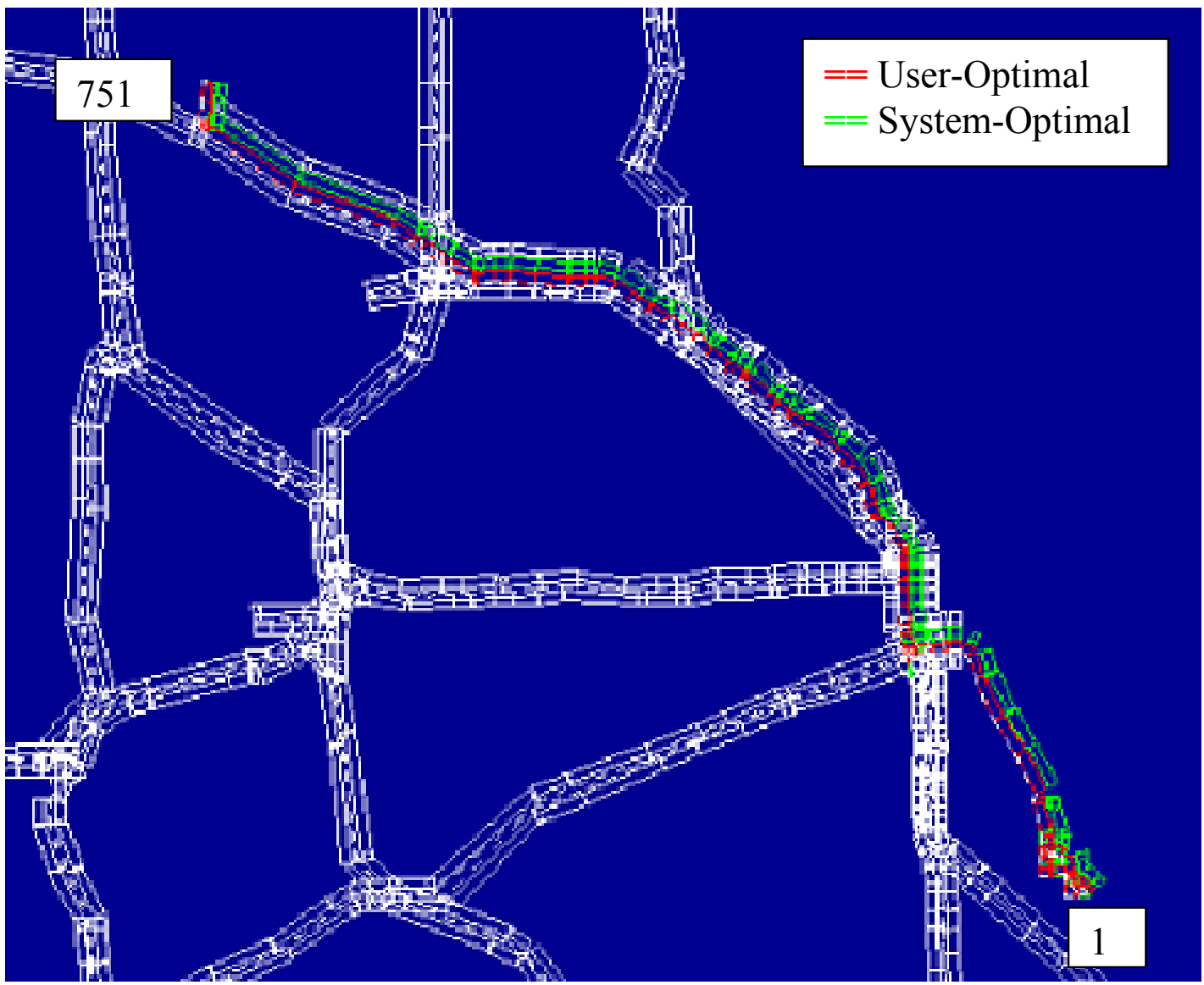

Figure 4: Links from zone 1 to zone 751 in the UO and SO solutions

In preparing these maps, the EMME/2 software system (INRO, 2004) was used to display the links that comprise the user-optimal and system-optimal routes between a single OD pair on a base map showing expressway and arterial links as white dashes. The Origin-Based Assignment algorithm was used to solve the two route choice problems. Each link that was included in any route was coloured red for user-optimal and green for system-optimal. In the case of a few routes, the coloured links clearly delineate the routes, but of course not the route flows, which are not unique in any case. In some maps, the coloured links illustrate how the route flows divide repeatedly in route patterns that are combinatorial in nature.

In Figure 4 links connecting zone 1 to zone 751 are shown for the user-optimal (red) and system-optimal (green) solutions. The travel times are 73.9 and 60.2 minutes respectively. The number of routes with flow in each solution is less than ten in each case. 
The SO solution has a substantially lower travel time because UO flows between many OD pairs using the heavily congested freeway from the central business district to the northwest are shifted to arterial links in the SO solution. For this case the routes for the two solutions are quite similar.

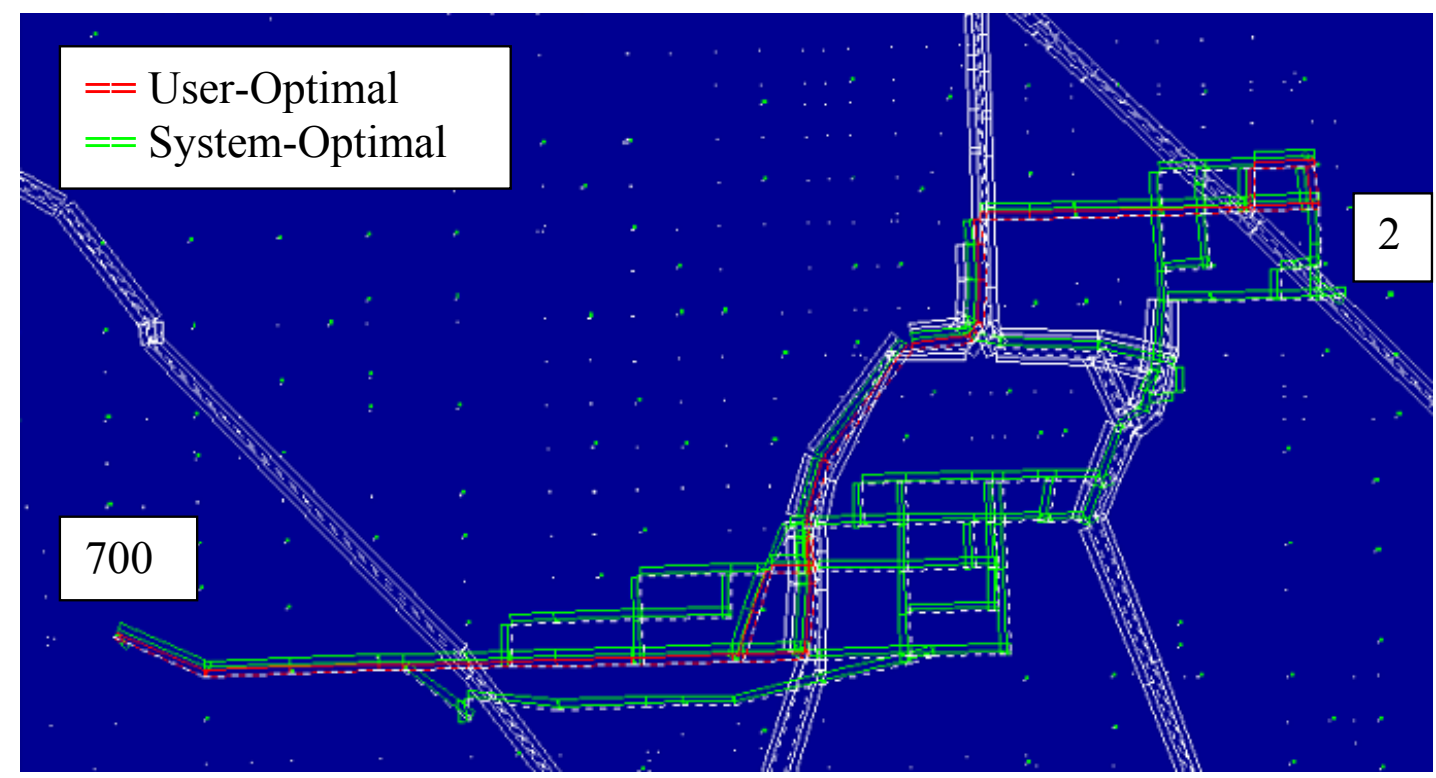

Figure 5: Links from zone 2 to zone 700 in the UO and SO solutions

Figure 5 shows a rather different situation for routes from zone 2 to zone 700 ; the UO travel time is 36.1 minutes, whereas the mean of the SO travel times is now 39.0 minutes. Although the number of UO routes is quite small, one can see from the figure that the number of SO routes is large, well over 1,000, as a result of combinatorial effects. Also, in this case the UO routes follow a freeway, while the SO routes follow arterials.

Figures 6 and 7 show a more complex situation for routes from zone 9 on the south shore of Lake Michigan to zone 292 in the far north side of Chicago (West Rogers Park). All routes, both user-optimal and system-optimal, utilize the Lake Shore Drive between its southern and northern termini. The user-optimal travel time is 67.0 minutes, and the mean system-optimal travel time is 67.5 minutes, so the travel times are essentially the same.

The number of distinct routes of each type is in the tens of thousands for each solution for a fixed trip table. In Figure 7 for the routes using arterial routes north of the northern terminus of Lake Shore Drive, the combinatorial pattern of both UO and SO routes can be plainly seen. A similar pattern found to the south of the southern terminus may be seen in Figure 6. The reader should keep in mind that for each OD pair all UO routes formed from the red links have equal travel times, and all SO routes formed from the green links have equal marginal travel times. 


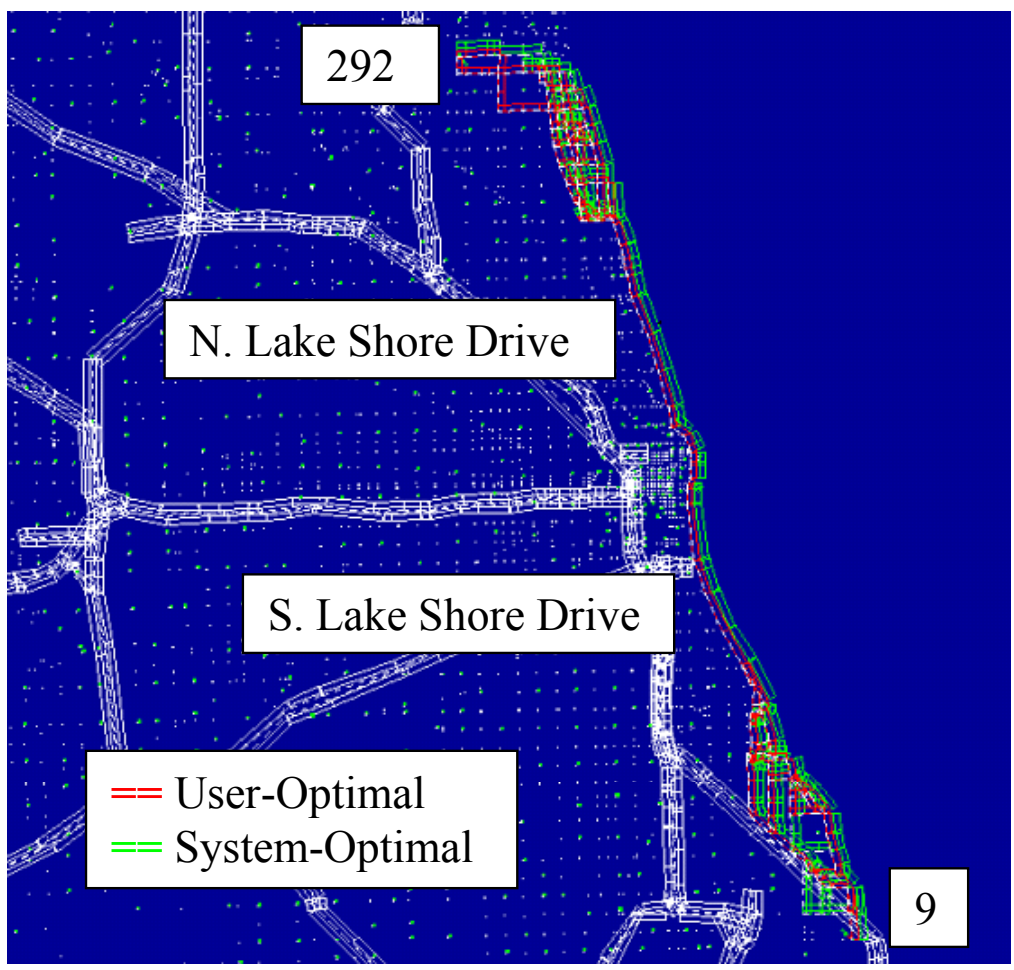

Figure 6: Links from zone 9 to zone 292 in the UO and SO solutions

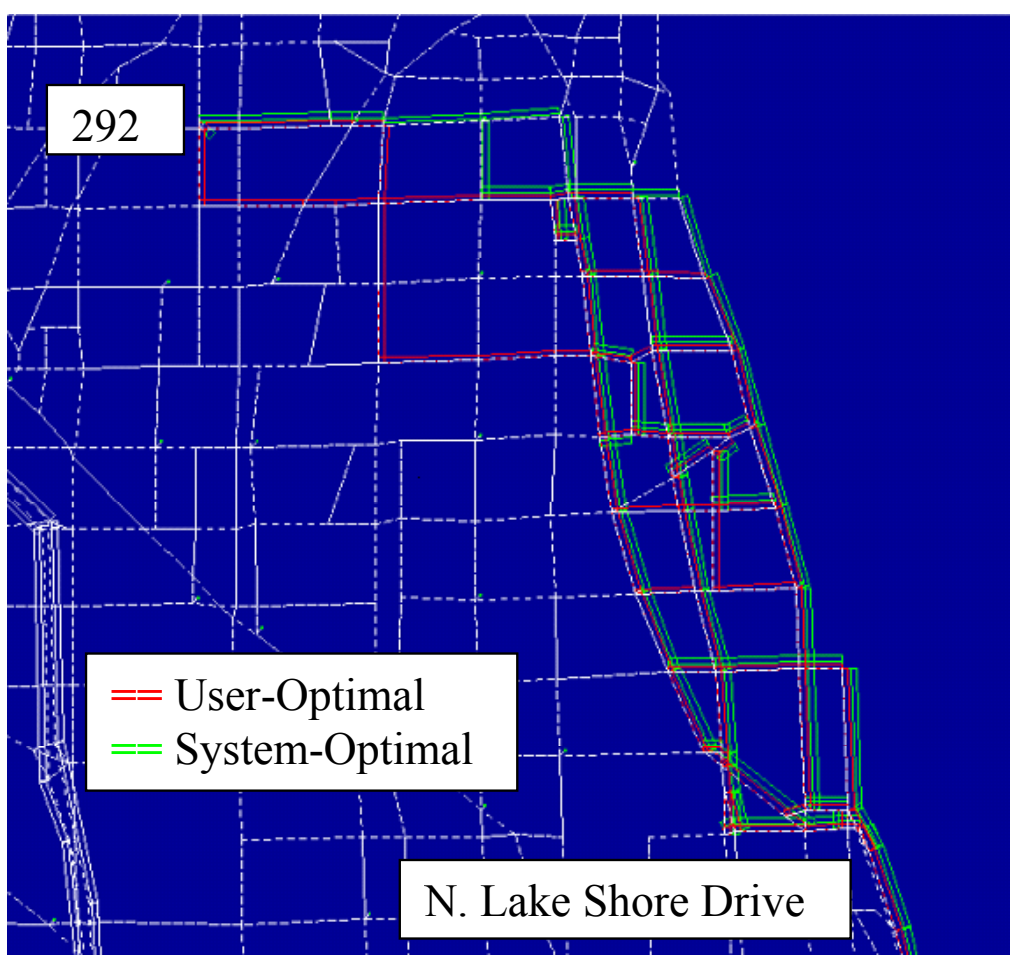

Figure 7: Links from N. Lake Shore Drive to zone 292 in the UO and SO solutions 


\section{Conclusions}

The findings presented here show the possible effects of shifting route and link flows from a behaviourally based user-optimal solution to the travel time minimizing system-optimal solution for a large, congested road network. As noted in the description of the solution method, the assumptions underlying the analysis are simplified and strong, but correspond to those widely applied in urban transportation planning. Shifting flows to the systemoptimal solution saves $5 \%$ of total travel time with an increase of $1 \%$ in travel distance.

The route choices found for individual OD pairs show that there are losers as well as winners in such a shift in route choice. Some travellers are worse off in the system-optimal solution, in some cases substantially so. To investigate this finding further, other variables than travel time should be included in the generalized cost function.

The results also show large numbers of routes for selected OD pairs for these solutions with a fixed trip table. Recent results for the variable demand case show substantially fewer routes than found in the results presented here. Ongoing research is exploring and analysing additional properties of this remarkable model.

\section{$6 \quad$ References}

Bar-Gera, H. (2002) "Origin-based Algorithms for the Traffic Assignment Problem," Transportation Science, 36: 398-417.

Beckmann, M., C. B. McGuire and C. B. Winsten (1956) Studies in the Economics of Transportation. Yale University Press: New Haven.

Boyce, D. and H. Bar-Gera (2003) "Validation of Urban Travel Forecasting Models Combining Origin-Destination, Mode and Route Choices," Journal of Regional Science, 43: 517-540.

INRO Consultants, Inc. (2004) EMME/2. http://www.inro.ca/

McDonald, J. F., E. L. d'Ouville and L. N. Liu (1999) Economics of Urban Highway Congestion and Pricing. Kluwer: Boston.

Patriksson, M. (1994) The Traffic Assignment Problem - Models and Methods. VSP: Utrecht.

Small, K. A. (1992) Urban Transportation Economics. Harwood Academic Publishers: Chur, Switzerland.

J. G. Wardrop (1952) "Some Theoretical Aspects of Road Traffic Research," Proceedings of the Institution of Civil Engineers, Part II, 1: 325-378.

Xiong, Q. (2002) Comparison of User-Optimal and System-Optimal Traffic Assignments for the Chicago Regional Road System, M.S. Thesis, University of Illinois at Chicago. 